
\title{
28 Research Square \\ Is Follow-up Needed for Infants with Graf Type I Hip at Screening?
}

Shohei Matsubayashi ( $\square$ bayazarov@gmail.com )

Graduate School of Biomedical Sciences https://orcid.org/0000-0003-2929-4594

\section{Hiroki Matsuzaki}

Graduate School of Biomedical Sciences, Nagasaki University

\section{Ritsu Tsujimoto}

Graduate School of Biomedical Sciences, Nagasaki University

\section{Makoto Osaki}

Nagasaki University School of Medicine Graduate School of Biomedical Sciences Nagasaki University

\section{Research article}

Keywords: Developmental dysplasia of the hip, Ultrasounds, Graf Type I, Acetabular dysplasia

Posted Date: November 23rd, 2020

DOl: https://doi.org/10.21203/rs.3.rs-111905/v1

License: (9) This work is licensed under a Creative Commons Attribution 4.0 International License. Read Full License 


\section{Abstract}

Background: The purpose was to determine if follow-up of infants with Graf Type I hip at screening is required.

Methods: Overall, 526 hips in 263 infants (61 males and 202 females) born between April 2015 and March 2018 who underwent primary screening for hip dislocation and presented to our department for secondary hip dislocation examination were included. Among these, 250 (59 males and 191 females) infants had bilateral Graf Type I hips for a total of 500 hips. Ultrasound results and physical examination at the initial visit and after 3 months were compared. X-rays were taken at the age of 1 year, and the proportion of infants with an acetabular index of $30^{\circ}$ or greater was evaluated.

Results: There were 0 hips that deteriorated from Graf Type I to II or higher among the infants. There were also 25 infants with abduction limitations in flexion at the initial visit, which was still present in 3 out of 23 infants 3 months later. There were 49 infants with asymmetry of the femoral or inguinal skin creases at the initial visit, which was still present in 9 out of 48 infants 3 months later. At the age of 1 year, an acetabular index of $30^{\circ}$ or greater was observed in 35 out of 402 hips (8.7\%).

Conclusion: Ultrasounds at 3 months later were not required in any of the infants with Graf Type I hip at initial diagnosis. Some infants were diagnosed with acetabular dysplasia at the age of 1 year.

\section{Introduction}

The Japanese Pediatric Orthopaedic Association publication 'Guide for hip dislocations in pediatric health examinations' lists five criteria: (1) limitations of abduction in flexion, (2) asymmetry of the femoral or inguinal skin creases, (3) family history, (4) female infants, and (5) breech delivery. The guidance recommends that those with either (1) present or meeting two or more of criterion (2) to (5) be referred for a secondary examination for hip dislocation [1]. Infant and toddler health examinations are conducted by paediatricians in Japan at 4 months of age. Thus, a primary examination for hip dislocation is performed, and the paediatric patient is referred to an orthopaedic surgeon for secondary examination of hip dislocation in accordance with the aforementioned guidance. Physical examinations, ultrasounds and $x$-ray examinations are carried out during the secondary hip dislocation examination [1].

It is clear that treatment and follow-up are necessary if acetabular dysplasia, subluxation or dislocation is observed in the secondary hip dislocation examination. However, in the absence of abnormalities, it is not known whether follow-up is necessary. There are two reasons for this. First, doctors cannot rule out the possibility of subsequent onset of acetabular dysplasia, subluxation or dislocation even if results are normal at the initial visit. Second, the cause of hip osteoarthritis in $81 \%$ of Japanese patients is acetabular dysplasia, $72 \%$ of whom are not previously treated for developmental dysplasia of the hip (DDH) [2]. If acetabular dysplasia that causes hip osteoarthritis in the future can be identified in the secondary hip dislocation examination, early treatment interventions, such as those implemented through Salter's pelvic osteotomy, may be possible. 


\section{Methods}

Overall, 526 hips in 263 infants (61 males and 202 females) born between April 2015 and March 2018 who underwent primary screening for hip dislocation and who presented to our department for secondary hip dislocation examinations were included. Medical interviews, physical examinations and ultrasounds were performed for all paediatric patients. Ultrasounds were performed using Graf grading [3]. Graf Type II or higher hip dislocation was determined as DDH.

The reasons for referral to our department for secondary hip dislocation examination were investigated. Infants who were determined by a paediatrician to have limitations of abduction in flexion and asymmetry of the femoral or inguinal skin creases were also assessed for these same parameters when seen by an orthopaedic specialist. The angle from the floor was set to be $20^{\circ}$ or greater for limitations of abduction in flexion.

There were 500 Graf Type I hips in 250 patients (59 males and 191 females). Ultrasound results were compared between the initial visit and 3 months later for infants with Graf Type I hip dislocation. An evaluation of whether or not there were improvements in limitations of abduction in flexion and femoral or inguinal skin crease asymmetry present at the initial visit was also performed. Additionally, $x$-rays were taken at the age of 1 year, and the proportion of infants with an acetabular index of $30^{\circ}$ or greater was evaluated.

\section{Results}

There were 9517 infants born in Nagasaki City from April 2015 to March 2018. Among these, 9251 infants were subjected to primary medical examination for hip dislocation in Nagasaki City. Among the 9251 infants, 488 (5.3\%) were referred for secondary hip dislocation examination. From these 488, 263 infants were taken to our department, and the rest were taken to other clinics (Table 1). The mean age was 4.6 months at the time of initial visit at our department. Graf Type II or higher hip dislocation was observed in $5.0 \%$ of infants (Table 2 ) and all were unilateral.

Table 1

Number referred for secondary hip dislocation examination among births between April 2015 and Mar 2018

\begin{tabular}{|ll|}
\hline & $\mathbf{n}$ \\
\hline Number of births & 9.517 \\
\hline Primary hip dislocation screening & 9.251 \\
\hline Secondary hip dislocation examination & 488 \\
\hline Seen at our department & 263 \\
\hline
\end{tabular}


Table 2

Graf classification

\begin{tabular}{|ll|}
\hline Type & $\mathbf{n ( \% )}$ \\
\hline I & $250(95.1)$ \\
\hline II & $9(3.4)$ \\
III & $2(0.8)$ \\
IV & $2(0.8)$ \\
\hline
\end{tabular}

Among infants with Graf Type I at initial visit, limitations of abduction in flexion was the most common reason for referral for a secondary hip dislocation examination (Table 3). The most common reason other than limitations of abduction in flexion alone was a combination of being a female infant and having asymmetry of the femoral or inguinal skin creases (Table IV).

Table 3

Reasons for referral for secondary hip dislocation examination

\begin{tabular}{|ll|}
\hline Reason for referral & $\mathbf{n}(\%)$ \\
\hline Limitations of abduction in flexion & $124(49.6)$ \\
\hline Asymmetry of skin creases & $82(32.8)$ \\
\hline Family history & $37(14.8)$ \\
\hline Female infant & $185(74.0)$ \\
\hline Breech delivery & $27(10.8)$ \\
\hline
\end{tabular}

Table 4

Combination of reasons other than limitations of abduction in flexion alone

\begin{tabular}{|ll|}
\hline Reasons for referral & $\mathbf{n}(\%)$ \\
\hline Female infant + asymmetry of skin creases & $75(30.0)$ \\
\hline Female infant + family history & $32(12.8)$ \\
\hline Female infant + breech delivery & $23(9.2)$ \\
\hline Asymmetry of skin creases + family history & $9(3.6)$ \\
\hline Breech delivery + family history & $5(2.0)$ \\
\hline Asymmetry of skin creases + breech delivery & $2(0.8)$ \\
\hline
\end{tabular}


Among infants with Graf Type I at initial visit, 20 out of 124 (16.1\%) actually had limitations of abduction in flexion at the time of the evaluation by an orthopaedic specialist. Femoral or inguinal skin crease asymmetry was confirmed in 20 out of $82(24.4 \%)$ infants.

The follow-up rate was $93.2 \%$ in 233 infants initially diagnosed with Graf Type I who were seen 3 months later. None of the hips worsened from Graf Type I to Graf Type II or higher after 3 months. There were 25 infants with limitations of abduction in flexion at the initial visit, and 23 were seen after 3 months, with limitations of abduction in flexion remaining in 2 infants. There was asymmetry of the femoral or inguinal skin creases at the initial visit in 49 infants, and asymmetry of the femoral or inguinal skin creases remained in 9 out of 48 infants seen 3 months later.

There were 201 infants diagnosed with Graf Type I at the initial visit who were seen at the age of 1 year, with a follow-up rate of $76.4 \%$. At the age of 1 year, the mean acetabular index was $24.1^{\circ}\left(22.7^{\circ}\right.$ in males and $24.5^{\circ}$ in females). An acetabular index of $30^{\circ}$ or greater was observed in 35 out of 402 hips (8.7\%).

\section{Discussion}

Screening for DDH appears to be performed during the neonatal period in Germany and Australia $[4,5]$. In Japan, it was reported in the article by Tanabe et al. in 1972 [6]. A neonatal examination using the click test was carried out. Despite the absence of abnormalities during the neonatal period, there were a number of paediatric patients in whom DDH was later diagnosed, indicating that it is of little significance to diagnose during the neonatal period. Instead, they recommended to perform screening at 3 months of age, when a diagnosis can easily be made. In Japan, the incidence of DDH was 1.1-3.5\% prior to 1965; however, Yamamuro et al. reported that the incidence of DDH decreased to $0.2 \%$ or less after a national campaign was launched to provide guidance on holding newborn babies and putting on diapers in 1975 [7]. Based on these changes, a policy to carry out preventive measures for hip dislocation, perform hip dislocation examinations at 3 months of age, and to perform treatment if there is DDH to all infants in Japan was established in the late 1970s. Subsequently, the conduct of hip dislocation examinations was also changed to 4 months of age since the timing of infant health examinations by paediatricians changed from 3 months to 4 months after birth.

Hattori et al. performed a nationwide Japanese survey via a multi-centre study. There were 1295 patients with DDH from 2011 to 2013 of whom 119 (15\%) were diagnosed at the age of 1 year or older. An additional 36 patients (3\%) were diagnosed at the age of 3 years or older [8]. This report raised the issue of delayed DDH diagnosis in Japan. The Japanese Pediatric Orthopaedic Association released publication, the 'Guide for hip dislocations in pediatric health examinations,' on their webpage in 2014 [1]. Secondary hip dislocation examinations based on this publication were started from 2015 in Nagasaki City, although secondary hip dislocation examinations had already been carried out at that point publication.

The Japanese Pediatric Orthopaedic Association's publication was prepared based on techniques referred to as the Matsudo method. Shinohara performed x-rays on all patients during infancy. The results were 
evaluated in detail and risk factors for DDH were extracted. In 1974, Shinohara developed a method for selectively x-raying infants with risk factors. It is called the Matsudo method since it was carried out in Matsudo city [9]. The results were reported in 2014 by Shinada. During the 41-year period, 196,643 infants were screened, and 10 were reported to have delayed diagnoses [10].

Limitations of abduction in flexion were the most frequent reason for referral to our department for secondary hip dislocation examinations (Table III). However, only 20 out of the 124 infants $(16.1 \%)$ actually had limitations of abduction in flexion. Since it is the paediatrician who performs the primary screening for hip dislocation, the cause may be that accuracy decreases when compared to the screening performed by paediatric orthopaedic specialists. Additionally, since there is a time lag from the primary screening for hip dislocation to the secondary hip dislocation examination, improvements may have occurred during that time.

Asymmetry of femoral or inguinal skin creases was the second most frequent reason for referral to our department for secondary hip dislocation examination. Asymmetry was actually confirmed in only 20 out of 82 infants (24.4\%). This rate may have been due to the fact that asymmetry was evaluated with hips extended and flexed and determined to be positive if observed in both or one as stated in the publication. We believe that femoral or inguinal skin crease asymmetry in a hip extended position has no pathological significance and therefore should not be identified as such. As Anderton et al. [11] have suggested, isolated asymmetrical skin creases are a not reliable clinical sign in the diagnosis of pathological DDH and we agree with this stance. Another common reason for being referred to our department was a combination of being a female infant and having asymmetry of the femoral or inguinal skin creases (Table IV). In the future, revisions to the Japanese Pediatric Orthopaedic Association's publication may be necessary.

None of the infants diagnosed with Graf Type I at 4 months of age worsened from Graf Type I to Graf Type II or higher at 3 months. Thus, a second ultrasound is not necessary in infants with Graf Type I hip. Biedermann et al. stated that $99.6 \%$ of infants with Graf Type I hip at 1 month of age had Graf Type I at 3 months of age [5]. This suggests that $0.4 \%$ of infants diagnosed at 1 month of age may worsen thereafter. The mean age at initial visit in our study was 4.6 months. We believe that the hips of infants at this time are already stable. Even if there are tentative limitations of abduction in flexion, the belief is that infants diagnosed with Graf Type I at 4 months of age will not worsen in terms of Graf classification. However, even with guidance provided on holding infants, limitations of abduction in flexion and femoral or inguinal skin crease asymmetry may remain 3 months later.

In a 1975 report, Yamamuro et al. stated that the mean acetabular index at the age of 1 year was $24.3^{\circ}$ in males and $23.1^{\circ}$ in females, which did not differ substantially from our results [12]. Additionally, the confidence limit for the acetabular index at $5 \%$ risk was $30.8^{\circ}$ in females and $30.5^{\circ}$ in males at the age of 1 year [12]. In our results, the acetabular index was $30^{\circ}$ at the age of 1 year in $7.7 \%$ of the total. Dornacher et al. noted that there were no significant correlations between Graf classification and radiological outcomes at follow-up [13]. In some infants diagnosed with Graf Type I hip dislocation at 4 months of 
age, acetabular dysplasia was diagnosed at the age of 1 year, and $x$-rays are considered to be required at least once.

\section{Conclusion}

None of the infants with Graf Type I hip at 4 months of age worsened 3 months later; thus, a second ultrasound is not necessary. However, limitations of abduction in flexion and femoral or inguinal skin crease asymmetry may remain 3 months later. In addition, there were infants diagnosed with acetabular dysplasia at the age of 1 year, and $\mathrm{x}$-rays are considered necessary.

\section{Abbreviations}

DDH Developmental Dysplasia of the Hip

\section{Declarations}

\section{Funding}

N/A

\section{Conflict of interest}

The authors declare that they have no financial or personal conflicts of interests that could have influenced this work.

\section{Ethics approval}

All procedures performed in this study were in accordance with the standards of the Ethics Committee of Nagasaki University Graduate School of Biomedical Sciences (approval number: 20032322).

\section{Consent to participate}

This is a retrospective study, so no consent to participate was obtained.

\section{Consent for publication}

This is a retrospective study, so no consent for publication was obtained.

\section{Availability of data and material}

This presentation data only

Code availability

$\mathrm{N} / \mathrm{A}$ 


\section{Authors' contributions}

The following authors contributed to conception and design Shohei Matsubayashi, Hiroki Matsuzaki, Ritsu Tsujimoto and Makoto Osaki. Analysis and Interpretation of the data was performed by Shohei Matsubayashi, Hiroki Matsuzaki and Ritsu Tsujimoto. All authors participated in drafting of the manuscript. Finally all authors have received and approved the final version of the manuscript as submitted.

\section{References}

1. The Japanese Pediatric Orthopaedic Association publication 'The Guide for hip dislocations in pediatric health examinations.' http://www.jpoa.org/

2. Jingushi S, Ohfuji S, Sofue M et al. Multi-institutional epidemiological study regarding osteoarthritis of the hip in Japan. J Orthop Sci. 2010;15:626-631.

3. Graf R. Classification of hip joint dysplasia by means of sonography. Arch Orthop Trauma Surg. 1984;102:248-255.

4. Farr S, Grill F, Müller D. Wann ist der optimale Zeitpunkt für ein sonographisches Hüftscreening? Orthopäde. 2008;37:532-540.

5. Biedermann R, Riccabona J, Giesinger JM. Results of universal ultrasound screening for developmental dysplasia of the hip: a prospective follow-up of 28092 consecutive infants. Bone Joint J. 2018;100-B(10):1399-1404.

6. Tanabe G, Kotakemori K, Miyake Y et al. Early diagnosis of congenital dislocation of the hip. Acta orthop Scand. 1972;43:511-522.

7. Yamamuro T, Ishida K. Recent advances in the prevention, early diagnosis, and treatment of congenital dislocation of the hip in Japan. Clin Orthop Relat Res. 1984;184:34-40.

8. Hattori T, Inaba Y, Ichinohe S et al. The epidemiology of developmental dysplasia of the hip in Japan: Findings from a nationwide multi-center survey. J Orthop Sci. 2017;22:121-126.

9. Shinohara $\mathrm{H}$, Tsuchiya $\mathrm{K}$, Tateno $\mathrm{Y}$ et al. A new method of screening for congenital hip dislocation in infants considering gonadal exposure to radiation in the population [article in Japanese]. RINSHO SEIKEIGEKA (Clinical Orthopaedic Surgery). 1974;9(3):203-211.

10. Shinada T, lida T, Kawamoto $T$ et al. Current status and future mass screening for congenital dislocation of the hip in Matsudo city. Seikei Geka. 2014;65(10):1017-1022.

11. Anderton MJ, Hastie GR, Paton RW. The positive predictive value of asymmetrical skin creases in the diagnosis of pathological developmental dysplasia of the hip. Bone Joint J. 2018;100-B(5):675-679.

12. Yamamuro T, Chene $\mathrm{SH}$. A radiological study on the development of the hip joint in normal infants. $J$ Jap Orthop Ass. 1975;49:421-439.

13. Dornacher $\mathrm{D}$, Cakir $\mathrm{B}$, Reichel $\mathrm{H}$ et al. Early radiological outcome of ultrasound monitoring in infants with developmental dysplasia of the hips. J Ped Orthop B. 2010;19(1):27-31. 